ARTIGO ORIGINAL

\title{
Variabilidade genética em população base de Eucalyptus viminalis em idade juvenil
}

\section{Genetic variability in a juvenile base population of Eucalyptus viminalis}

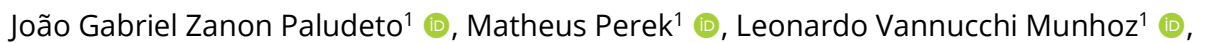 \\ Jéssica Ritchele Moura dos Santos ${ }^{1}$ [D, Vagner Alex Pesck ${ }^{1}$ (i), Evandro Vagner Tambarussi ${ }^{1}$ (])
}

${ }^{1}$ Universidade Estadual do Centro-Oeste - UNICENTRO, Irati, PR, Brasil

Como citar: Paludeto, J. G. Z., Perek, M., Munhoz, L. V., Santos, J. R. M., Pesck, V. A., \& Tambarussi, E. V. (2020) Variabilidade genética em população base de Eucalyptus viminalis em idade juvenil. Scientia Forestalis, 48(126), e3081. https://doi.org/10.18671/scifor.v48n126.07

\section{Resumo}

O presente estudo teve como objetivo caracterizar a variabilidade genética existente em uma população base de $E$. viminalis em idade juvenil. O ensaio foi instalado em blocos ao acaso, contendo 63 progênies distribuídas em 17 procedências. Foram mensurados os caracteres diâmetro a altura do colo (DAC, mm) aos sete meses de idade e altura total (ALT, cm) aos quatro (ALT4), cinco (ALT5), seis (ALT6) e sete (ALT7) meses de idade. A herdabilidade no sentido restrito $\left(\hat{h}_{a}^{2}\right)$ foi baixa para $\operatorname{DAC}(0,15)$, média para altura aos seis meses $(\operatorname{ALT} 6 ; 0,29)$ e sete meses (ALT7; 0,27) e alta para altura aos quatro meses (ALT4; 0,63 ) e cinco meses de idade (ALT5; 0,48$)$. A diferenciação genética entre progênies dentro de procedências $\left(\hat{Q}_{S T_{\text {prog } / \text { proc }}}\right.$ ) e entre procedências $\left(\hat{Q}_{S T_{\text {proc }}}\right)$ para DAC foram baixas e mostraram que apenas $7 \%$ da variação genética total está concentrada nestes níveis. Ao passar dos meses, para o caractere ALT, percebe-se que há maior variação genética alocada ao nível de procedências (ALT7; 11,2\%) do que entre progênies dentro de procedências (ALT7; 8,1\%). Os valores de coeficiente de variação genética $\left(C \hat{V}_{g} \%\right.$ ) variaram de 7,03\% (DAC) a 11,63\% (ALT4). O tamanho efetivo $\left(N_{e}\right)$ estimado da população foi de até 149 . Os resultados da presente pesquisa, apontam que há variabilidade genética em idade precoce na população base em estudo.

Palavras-chave: Parâmetros genéticos; Divergência genética; Procedências; Tamanho Efetivo.

\begin{abstract}
The present study aimed to assess the genetic variability of a Eucalyptus viminalis juvenile base population. The installed experiment consisted of randomized blocks containing 63 progenies distributed across 17 provenances. The root collar diameter (DAC, $\mathrm{mm}$ ) was measured at seven months of age and total height (ALT, cm) was assessed at four (ALT4), five (ALT5), six (ALT6), and seven (ALT7) months. The narrow-sense heritability $\left(\hat{h}_{a}^{2}\right)$ was low for DAC $(0.15)$, medium for height at six and seven months (ALT6, 0.29; ALT7, 0.27) and high for height at four and five months of age (ALT4, 0.63; ALT5, 0.48). For DAC, the genetic differentiation between progenies within provenances $\left(\hat{Q}_{S T_{\text {prog } / \text { proc }}}\right)$ and among provenances ( $\hat{Q}_{S T_{\text {proc }}}$ ) was low and showed that only $7 \%$ of the total genetic variation is concentrated at the progeny and provenance levels. At the end of the study period, the results showed more genetic variation for height at the provenance level (ALT7, 11.2\%) than among progenies within provenances (ALT7, 8.1\%). The coefficient of genetic variation $\left(C \hat{V}_{g}(\%)\right)$ varied from $7.03 \%(D A C)$ to $11.63 \%$ (ALT4). The estimated effective population size $\left(N_{e}\right)$ reached up to 149 . The results of the present study indicate that genetic variability exists from an early age in the studied base population.
\end{abstract} Keywords: Genetic parameters; Genetic divergence; Provenances; Effective population Size.

Fonte de financiamento: Nenhuma.

Conflito de interesse: Nada a declarar.

Autor correspondente: tambarussi@gmail.com

Recebido: 18 setembro 2018.

Aceito: 17 junho 2019.

Editor: Francides Gomes Silva Júnior

(c) (1) Este é um artigo publicado em acesso aberto (Open Access) sob a licença Creative Commons Attribution, que permite uso, distribuição e reprodução cc. em qualquer meio, sem restrições desde que o trabalho original seja corretamente citado. 


\section{INTRODUÇÃO}

O gênero Eucalyptus pertence à família Myrtaceae, sendo reconhecidas cerca de 800 espécies, variedades e híbridos (Flores et al., 2016). No Brasil, as florestas plantadas com o gênero Eucalyptus destacam-se no setor florestal brasileiro por apresentar grande adaptação, rápido crescimento, elevada produtividade e diversas finalidades (Tambarussi et al., 2017). O Eucalyptus viminalis Labill é uma espécie adaptada a áreas sujeitas a geadas, especialmente na Região Sul do Brasil (Higa \& Carvalho, 1990) e possui um potencial madeireiro destinado principalmente para lenha e produção de carvão (Sturion, 1993).

O E. viminallis vem sendo utilizado em processos de hibridação (Santos et al., 2013) e populações puras da espécie vem se tornando cada vez mais raras. O melhoramento florestal visa o aumento da produtividade aliado a qualidade dos recursos florestais a cada ciclo de seleção, preservando a população base para futuros programas de melhoramento genético. Além disso, o melhoramento genético pode proporcionar melhor adaptação das espécies, tolerância a pragas e doenças, melhorias nas qualidades físicas e mecânicas da madeira e a manutenção da variabilidade genética (Mori, 1988; Pigato \& Lopes, 2001).

Para obter uma espécie melhorada, é preciso conhecer o germoplasma disponível, a sua diversidade genética entre e dentro das espécies e entre e dentro de populações, e identificar os genótipos com as características desejáveis para a utilização em programas de melhoramento genético. Além disso, é necessário conhecer os métodos de seleção e visando identificar o material genético superior para cada ambiente.

As estimativas dos parâmetros genéticos são de grande ajuda para os melhoristas (Moraes et al., 2011; Miranda et al., 2013). Atualmente, a metodologia de modelos mistos REML/BLUP é o procedimento bastante utilizado na avaliação genética no melhoramento florestal, devido a características como correção simultânea dos dados para os efeitos ambientais, comparação de indivíduos no tempo e espaço, produção de resultados não viesados e maximização da acurácia de seleção (Resende, 2007). Esta metodologia é útil para dados desbalanceados, pois maximiza o ganho genético e a eficiência dos programas de melhoramento além de considerar o parentesco genético entre os indivíduos (Resende, 2007; Pires et al., 2011).

A diversidade genética é fundamental para definição de estratégias de conservação, manejo e melhoramento genético para uma espécie (Brandão et al., 2015; Duarte et al., 2015). Uma forma de auxiliar na manutenção da diversidade genética é a conservação ex situ que envolve a manutenção da variabilidade genética em condições artificiais, ou seja, fora do seu habitat natural (Sebbenn \& Ettori, 2001; Faleiro et al., 2011). A conservação ex situ auxilia tanto na preservação de espécies ameaçadas de extinção quanto em programas de melhoramento genético (Faleiro et al., 2011), e pode ser feita por meio de sementes, culturas de tecidos, coleções de plantas mantidas em campo, entre outros (Paiva \& Valois, 2001).

Dessa forma, o estudo teve como objetivo estimar parâmetros genéticos de caracteres juvenis capazes de expressar a variabilidade genética existente em uma população base de E. viminalis.

\section{MATERIAL E MÉTODOS}

\section{Área de Estudo}

Os dados foram coletados de uma população base de E. viminalis localizada na Universidade Estadual do Centro-Oeste (UNICENTRO) em Irati, Paraná (Figura 1).

A população base de $E$. viminalis faz parte do Programa Cooperativo de Melhoramento Florestal (PCMF) (Instituto de Pesquisas e Estudos Florestais, 2018). O objetivo da implantação destas populações é a conservação ex situ de um acervo de materiais genéticos em diversas regiões com condições edafoclimáticas distintas, viabilizando a identificação de materiais superiores e adaptados a estas condições (Silva et al., 2018). 


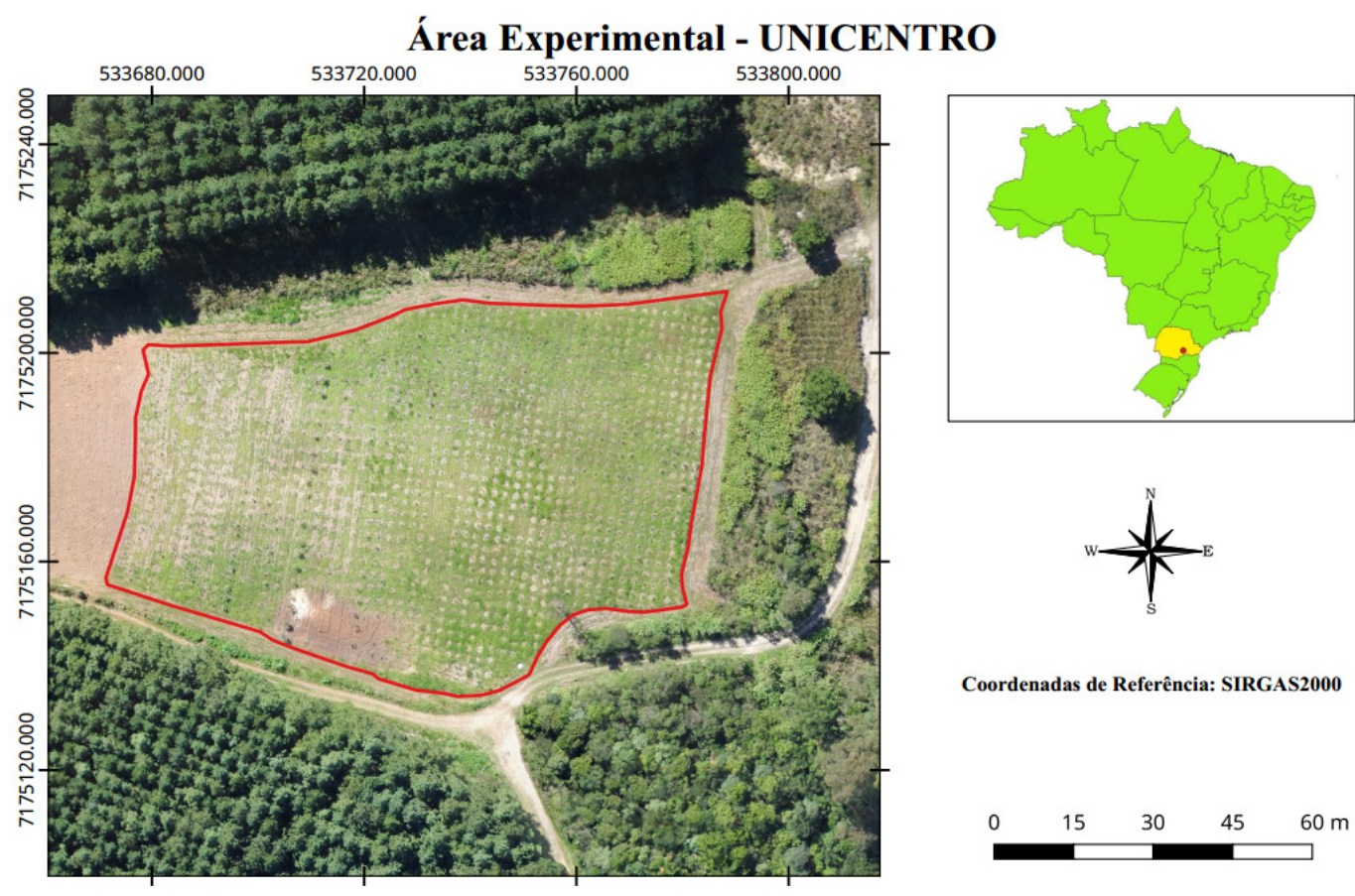

Figura 1. População base de E. viminalis situada na Universidade Estadual do Centro-Oeste no município de Irati, Paraná.

O clima da região é do tipo Cfb, ou seja, subtropical úmido sem estação seca, geadas severas e frequentes, com temperatura média do mês mais frio superior a $10^{\circ} \mathrm{C}$, e do mês mais quente, inferior a $22^{\circ} \mathrm{C}$ (Köppen \& Geiger, 1928). Segundo a base de dados (1963-2018) da Estação Meteorológica do Instituto Agronômico do Paraná (2019) situada em Fernandes Pinheiro, município situado a aproximadamente $15 \mathrm{~km}$ de Irati, a região apresenta média anual de precipitação de $1.609 \mathrm{~mm}$. As temperaturas médias dos meses mais quentes e mais frios são de respectivamente $24,0^{\circ} \mathrm{C}$ e $13^{\circ} \mathrm{C}$. Entretanto, durante todo seu funcionamento, a estação detectou temperatura máxima de $36,6^{\circ} \mathrm{C}(17 / 11 / 85)$ e mínima de $-5,7^{\circ} \mathrm{C}(18 / 07 / 75)$.

O experimento foi implantado no mês de dezembro de 2017 em blocos ao acaso. O ensaio é composto de três blocos, com 63 progênies pertencentes a 17 procedências (Silva et al., 2018, p. 96), dispostas em parcelas lineares de 3 plantas. As alturas das plantas $(A L T, \mathrm{~cm})$ foram mensuradas aos quatro (ALT4), cinco (ALT5), seis (ALT6) e sete (ALT7) meses de idade. Mensurou-se também o diâmetro do colo a $20 \mathrm{~cm}$ do solo (DAC, $\mathrm{mm}$ ) aos sete meses de idade.

O experimento foi analisado utilizando o pacote estatístico Ime4 (Bates et al., 2015), por meio do ambiente estatístico R (R Core Team, 2017), pelo seguinte modelo misto:

$y=X \beta+Z_{1} g+Z_{2} p+Z_{3} r g+e$

em que: $\boldsymbol{\beta}$ : vetor de efeitos fixos associados ao bloco; $\boldsymbol{g}$ : vetor de efeitos aleatórios associados às progênies; $\boldsymbol{p}$ : vetor de efeitos aleatórios associados às procedências; $\boldsymbol{r} \boldsymbol{g}$ : vetor de efeitos aleatórios associados à interação bloco x progênies; $\boldsymbol{e}$ : vetor de erros aleatórios.

A significância dos efeitos aleatórios do modelo foi constatada por meio da razão de verossimilhança (LRT) utilizando o teste de qui-quadrado com 1 grau de liberdade. Já a significância do efeito de bloco (fixo) foi testada de acordo com o teste $\mathrm{F}$ com aproximação de Satterthwaite (Satterthwaite, 1946). 


\section{Estimativas de parâmetros genéticos}

Considerando que o parentesco entre os indivíduos são meios-irmãos, obteve-se a variância genética aditiva $\left(\hat{\sigma}_{a}^{2}=4 \hat{\sigma}_{\text {prog }}^{2}\right)$. Os coeficientes de herdabilidades foram estimados ao nível individual no sentido restrito $\left(\hat{h}_{a}^{2}\right)$ e herdabilidade dentro de progênies $\left(\hat{h}_{d}^{2}\right)$ por meio das seguintes equações:

$$
\hat{h}_{a}^{2}=\frac{\hat{\sigma}_{a}^{2}}{\hat{\sigma}_{\text {prog }}^{2}+\hat{\sigma}_{\text {proc }}^{2}+\hat{\sigma}_{r g}^{2}+\hat{\sigma}_{e}^{2}} ; \hat{h}_{d}^{2}=\frac{3 \hat{\sigma}_{p r o g}^{2}}{\hat{\sigma}_{e}^{2}}
$$

em que: $\hat{\sigma}_{\text {prog }}^{2}$ : variância genotípica das progênies; $\hat{\sigma}_{\text {proc }}^{2}$ : variância das procedências; $\hat{\sigma}_{r g}^{2}$ : variância da interação entre bloco e progênie; $\hat{\sigma}_{e}^{2}$ : variância do erro residual total. As estimativas dos coeficientes de variação genética foram obtidas por $C \hat{V}_{g}(\%)=\frac{\sqrt{\hat{\sigma}_{p r o g}^{2}}}{\bar{x}} \cdot 100$, variação genética aditiva individual por $C \hat{V}_{g i}(\%)=\frac{\sqrt{\hat{\sigma}_{a}^{2}}}{\bar{x}} \cdot 100$ e ambiental $C \hat{V}_{e}(\%)=\frac{\sqrt{\hat{\sigma}_{e}^{2}}}{\bar{x}} \cdot 100$. Para amparar a seleção das melhores progênies foi estimada a acurácia seletiva $\hat{r}_{a a}=\sqrt{\hat{h}_{m}^{2}}$.Por meio de uma relação entre os coeficientes de variação, obtivemos o coeficiente de variação alélica $\left(\hat{c}=\frac{C \hat{V}_{g i}}{C \hat{V}_{g}}\right)$.

Para a estimativa das divergências genéticas entre as procedências $\left(\hat{Q}_{S T_{\text {proc }}}\right)$ e entre as progênies dentro de procedências $\left(\hat{Q}_{S T_{\text {prog } / \text { roro }}}\right)$ foram utilizados estimadores análogos aos de Spitze (1993):

$$
\hat{Q}_{S T_{\text {proc }}}=\frac{\hat{\sigma}_{\text {proc }}^{2}}{\hat{\sigma}_{e}^{2}+\hat{\sigma}_{\text {prog }}^{2}+\hat{\sigma}_{\text {proc }}^{2}} ; \hat{Q}_{S T \text { prog } / \text { proc }}=\frac{\hat{\sigma}_{\text {prog }}^{2}}{\hat{\sigma}_{e}^{2}+\hat{\sigma}_{\text {prog }}^{2}+\hat{\sigma}_{\text {proc }}^{2}}
$$

Foi realizada uma simulação de seleção individual nas intensidades de 30\%, 60\% e 90\% via metodologia BLUP, a qual serviu como base na estimativa do tamanho efetivo populacional $\left(N_{e}\right)$, que foi obtido de acordo com Resende (2002):

$$
N_{e}=\frac{4 N_{p} \bar{k}_{f}}{3+\bar{k}_{f}\left(\sigma_{k f}^{2} / \bar{k}_{f}\right)}
$$

em que $N_{p}$ : número de progênies selecionadas; $\bar{k}_{f}$ : média do número de indivíduos selecionados dentro das progênies; $\sigma_{k f}^{2}$ : variância do número de indivíduos selecionados por progênies.

\section{RESULTADOS E DISCUSSÃO}

A significância dos efeitos aleatórios do modelo para os dois caracteres, testada pela razão de verossimilhança (LRT) (Tabela 1), revelou não significância dos efeitos de progênie e procedência para o caractere DAC aos sete meses de idade. Para ALT, o efeito de progênie foi significativo apenas nas duas primeiras medições (ALT4 e ALT5). Já o efeito de procedência, foi significativo em todos os meses de avaliação, revelando a existência de elevada variabilidade no desempenho das procedências avaliadas. A interação entre bloco e progênie (Rep x Prog), a qual corresponde ao efeito ambiental, também foi significativa para os dois caracteres em todos os meses avaliados. 
Tabela 1 - Teste de razão da verossimilhança (LRT) para diâmetro a altura do colo (DAC, mm) aos sete meses de idade e altura total (ALT, $\mathrm{cm}$ ) de $E$. viminallis variando dos quatro aos sete meses de idade, dos efeitos de procedência (Proc), progênie (Prog) e interação entre bloco e progênie (Rep x Prog).

\begin{tabular}{cccc}
\hline \multirow{2}{*}{ Caractere } & \multicolumn{3}{c}{ Efeito } \\
\cline { 2 - 4 } & Prog & Proc & Rep $\times$ Prog \\
\hline DAC & $0,74^{\text {ns }}$ & $1,74^{\text {ns }}$ & $14,16^{* *}$ \\
ALT4 & $10,65^{* *}$ & $7,14^{* *}$ & $20,07^{* *}$ \\
ALT5 & $7,75^{* *}$ & $5,75^{*}$ & $7,19^{* *}$ \\
ALT6 & $2,70^{\text {ns }}$ & $5,33^{*}$ & $17,78^{* *}$ \\
ALT7 & $2,45^{\text {ns }}$ & $7,40^{* *}$ & $13,83^{* *}$ \\
\hline
\end{tabular}

** significativo a $1 \%$ de probabilidade; * significativo a $5 \%$ de probabilidade; ns não significativo.

Segundo a classificação de Pimentel-Gomes \& Garcia (2002), os valores do coeficiente de variação experimental ( $C \hat{V}$ \%) encontrados no presente estudo, são considerados médios $(22,22 \%$ a $23,09 \%)$ para ALT $(\mathrm{cm})$ e alto $(31,72 \%)$ para DAC $(\mathrm{mm})$ (Tabela 2). Entretanto, de acordo com Moraes et al. (2015), esta classificação não leva em consideração os diferentes fatores genéticos e ambientais e é comum em experimentos florestais a nível de campo a existência de $C_{\hat{V}} \%$ acima de $30 \%$.

Tabela 2 - Estimativa de componentes de variância e parâmetros genéticos de progênies e procedências de $E$. viminalis para os caracteres DAC $(\mathrm{mm})$ aos sete meses de idade e ALT $(\mathrm{cm})$ dos quatro aos sete meses de idade.

\begin{tabular}{cccccc}
\hline Parâmetro & DAC $(\mathbf{m m})$ & ALT4 $(\mathbf{c m})$ & ALT5 $(\mathbf{c m})$ & ALT6 $(\mathbf{c m})$ & ALT7 $(\mathbf{c m})$ \\
\hline$\hat{\sigma}_{\text {prog }}^{2}$ & 0,28 & 50,64 & 52,46 & 46,79 & 51,08 \\
$\hat{\sigma}_{r g}^{2}$ & 1,47 & 53,98 & 47,95 & 122,03 & 127,51 \\
$\hat{\sigma}_{\text {proc }}^{2}$ & 0,19 & 31,87 & 35,40 & 47,39 & 70,70 \\
$\hat{\sigma}_{e}^{2}$ & 5,79 & 184,86 & 303,43 & 429,78 & 504,69 \\
$\hat{\sigma}_{F}^{2}$ & 7,73 & 321,84 & 439,26 & 646,00 & 753,94 \\
$\hat{h}_{a}^{2}$ & 0,15 & 0,63 & 0,48 & 0,29 & 0,27 \\
$\hat{h}_{d}^{2}$ & 0,15 & 0,82 & 0,52 & 0,33 & 0,30 \\
$\hat{Q}_{S T_{\text {proc }}}$ & 0,04 & 0,119 & 0,091 & 0,090 & 0,112 \\
$\hat{Q}_{S T_{\text {prog } / \text { proc }}}$ & 0,03 & 0,189 & 0,130 & 0,089 & 0,081 \\
$C \hat{V}_{g}(\%)$ & 7,03 & 11,63 & 9,36 & 7,46 & 7,34 \\
$C \hat{V}_{g i}(\%)$ & 14,06 & 23,26 & 18,70 & 14,93 & 14,68 \\
$C \hat{V}_{e}(\%)$ & 31,72 & 22,22 & 22,52 & 22,61 & 23,09 \\
$\hat{c}$ & 2,0 & 2,0 & 2,0 & 2,0 & 2,0 \\
$\bar{x}$ & 7,58 & 61,16 & 77,32 & 91,89 & 97,32 \\
\hline
\end{tabular}

$\hat{\sigma}_{p r o g}^{2}$ : variância da progênie; $\hat{\sigma}_{r g}^{2}$ : variância da interação entre repetição e a progênie; $\hat{\sigma}_{\text {proc }}^{2}$ : variância da procedência; $\hat{\sigma}_{e}^{2}$ : variância residual (dentro); $\hat{\sigma}_{F}^{2}$ : variância fenotípica; $\hat{h}_{a}^{2}$ : herdabilidade no sentido restrito; $\hat{h}_{d}^{2}$ : herdabilidade dentro; $\hat{Q}_{S T_{\text {proc }}}$ : divergência genética entre procedências; $\hat{Q}_{S T_{\text {prog } / \text { proc }}}$ : divergência genética entre progênies dentro de procedências; $C \hat{V}_{g}(\%)$ : coeficiente de variação genética; $C \hat{V}_{g i}(\%)$ :coeficiente de variação genética individual; $C \hat{V}_{e}(\%)$ : coeficiente de variação experimental; $\hat{c}$ : coeficiente de variação alélica; $\bar{x}:$ média geral.

Na presente pesquisa, os valores de $C \hat{V}_{g}$ \% encontrados para os caracteres avaliados foram condizentes com os da literatura (Tabela 2). Tambarussi et al. (2017), encontraram valores de 
coeficiente de variação genética ( $C \hat{V}_{g} \%$ ) para Eucalyptus spp. aos três anos de idade que variaram de 2,65\% a 20,95\% para diâmetro a altura do peito, e de 1,83\% a 14,89\% para altura.

Para ALT, houve um decréscimo dos valores de $C \hat{V}_{g} \%$ ao longo dos meses avaliados (de 11,63\% para ALT4 até 7,34\% para ALT7). Como a variância entre progênies ( $\hat{\sigma}_{\text {prog }}^{2}$ ), não se alterou significativamente durante as medições, o decréscimo do $C \hat{V}_{g} \%$ ocorreu devido ao incremento na média $(\bar{x})$ do caractere, um comportamento esperado durante o crescimento inicial de um plantio. Apesar do decréscimo de $\hat{C V}_{g} \%$ nestas idades iniciais, alguns autores (Massaro et al., 2010; Tambarussi et al., 2017; Paludeto et al., 2017), relatam o aumento deste parâmetro com o avanço do tempo. Com o passar dos anos, uma maior expressão alélica do genótipo tende a aumentar a variância genética, à medida que a média tende a se estagnar.

$\mathrm{O} \hat{Q}_{S T}$ é originalmente um estimador da diferença genética representada pelas variâncias genéticas que influenciam a expressão fenotípica de um caráter dentro e entre populações, sendo bastante útil na mensuração da variabilidade genética de um material (Lande, 1992; Whitlock, 2008).

Para o DAC $(\mathrm{mm})$, as estimativas de divergência genética entre procedências $\left(\hat{Q}_{S T_{\text {proc }}}=0,04\right)$ e progênies dentro de procedência $\left(\hat{Q}_{S T_{\text {prog } / \text { proc }}}=0,03\right.$ ) foram baixas, e somadas, representam apenas $7 \%$ da variação genética total, o que significa, portanto, que $93 \%$ da variação fenotípica total está distribuída dentro de progênies para este caractere aos sete meses de idade. Este resultado é semelhante ao encontrado por Sebbenn et al. (2007) em seu estudo com progênies de Balfourodendron riedelianum aos 21 anos de idade; o autor concluiu que a maior parte da variação genética influente no fenótipo (95\%) estava alocada dentro de progênies.

O caractere $A L T(\mathrm{~cm})$, nos primeiros meses, possui divergência genética entre progênies dentro de procedências $\left(\hat{Q}_{S T_{\text {prog } / \text { roc }}}\right)$ superior à divergência genética entre procedências $\left(\hat{Q}_{S T_{\text {proc }}}\right)$. No entanto, percebe-se uma diminuição desta diferença com o passar dos meses, até o ponto em que $\hat{Q}_{S T_{\text {proc }}}(0,112 ; A L T 7)$ supera o valor de $\hat{Q}_{S T_{\text {prog proc }}}(0,081 ; A L T 7)$, significando que, para o este caractere, nesta idade, $11,2 \%$ da variância genética está alocada entre procedências e $8,1 \%$ entre progênies dentro procedências. Estes resultados diferem da afirmação de Konzen et al. (2017) acerca desta estimativa, os autores relatam que comumente para caracteres quantitativos em espécies florestais, a variância genética tende a estar distribuída de forma mais acentuada dentro de procedências. Entretanto, tal diferença pode estar associada à idade precoce de avaliação do presente estudo.

O coeficiente de variação alélica $(\hat{c})$ encontrado foi de 2,0 para todos os caracteres avaliados. Esta estimativa, quando igual a 1, indica que a variância genética entre procedências é nula, porém quando superior a unidade, demonstra que há variação genética dentro das procedências a ser explorada pelo melhoramento (Pires, 1996). O elevado valor deste parâmetro se atribui aos maiores valores de $C \hat{V}_{g i} \%$ encontrados os caracteres avaliados (variando de $14,68 \%$ até $23,26 \%$ para ALT e $14,06 \%$ para DAC) em relação ao $C \hat{V}_{g} \%$. O $C \hat{V}_{g i} \%$ está intimamente relacionado com o sucesso dos cruzamentos de um programa de melhoramento, pois quanto maior seu valor, maior $0 \hat{c}$, e como consequência, maior o sucesso na transmissão dos genes aditivos para a prole (Falconer \& MacKay, 1996).

Segundo a classificação de Resende (1995), os valores de $\hat{h}_{a}^{2}$ encontrados para ALT4 $(0,63)$ e $\operatorname{ALT5}(0,48)$ são considerados de alta e média magnitude, respectivamente, já para ALT6 $(0,29)$, ALT7 $(0,27)$ e DAC $(0,15)$ de média a baixas magnitudes. Estes altos valores de herdabilidade nos dois primeiros meses de avaliação, provavelmente não são condizentes com a realidade do material genético, pois os genótipos sofreram pouca influência ambiental, algo que exerce muita influência em características quantitativas. Isto pode ser visualizado na Tabela 2, onde percebe-se baixa alteração da variância genética $\left(\hat{\sigma}_{\text {prog }}^{2}\right)$, e alta variação do fenótipo ( $\hat{\sigma}_{F}^{2}$ ) ao longo dos meses de medição. 
Estes valores de $\hat{h}_{a}^{2}$ são considerados medianos e corroboram com os que são comumente encontrados na literatura, variando de 0,15 a 0,50, para populações iniciais de Eucaliptos (Resende, 2002). Em Eucalyptus urophylla avaliados com um ano de idade por Henriques et al. (2016), encontraram $\hat{h}_{a}^{2}$ baixas e intermediarias para DAP e ALT (0,10 e 0,29, respectivamente). Paludzyszyn Filho \& Santos (2011), avaliando Eucalyptus benthamii aos 20 meses pós plantio, obteve $\hat{h}_{a}^{2}$ de 0,40 para DAP. Sturion (1993), estudando E. viminalis aos 43 meses de idade, encontraram $\hat{h}_{a}^{2}$ intermediárias para DAP e $\operatorname{ALT}(0,20$ e 0,26, respectivamente).

As estimativas de herdabilidade dentro $\left(\hat{h}_{d}^{2}\right)$ para os dois caracteres, caso se desconsidere as duas primeiras medições da característica ALT, mostraram-se de média magnitude (0,33 e 0,30 para ALT6 e ALT7, respectivamente; 0,15 para DAC). Com relação aos valores de herdabilidade estimados, pode-se concluir que mesmo em idade precoce, há significante controle genético individual, entre e dentro de progênies.

A avaliação numérica das diferentes intensidades de seleção permitiu o entendimento do tamanho efetivo populacional ( $N_{e}$ ) que a população possui. Este parâmetro foi estimado apenas para o caractere ALT6. Para as intensidades de seleção de 30 e 60\%, foram encontrados valores de $N_{e}$ que variaram de 73,5 e 117,1. Porém, ao diminuir a intensidade, selecionando quase a totalidade do teste (90\%), foi encontrado um $N_{e}$ de 148,6. Para uma população base, é essencial a manutenção da variabilidade genética existente. Tamanhos efetivos na casa das centenas produzem segurança razoável contra a perda de alelos por efeito da deriva (Vencovsky, 1987).

\section{CONCLUSÕES}

A população base de $E$. viminalis implantada no município de Irati-PR possui variabilidade genética para os caracteres altura total $(A L T, c m)$ e diâmetro a altura do colo (DAC, mm), e retém tamanho efetivo suficiente para a conservação ex situ da população até as idades avaliadas.

\section{AGRADECIMENTOS}

Os autores agradecem ao Instituto de Pesquisas e Estudos Florestais (IPEF) pela disponibilização das sementes e informações sobre as procedências utilizadas na presente pesquisa. O professor Dr. Evandro V. Tambarussi agradece ao CNPq pela bolsa Produtividade (PQ-2).

\section{REFERÊNCIAS}

Bates, D., Mächler, M., Bolker, B., \& Walker, S. (2015). Fitting linear mixed-effects models using Ime4. Journal of Statistical Software, 67(1), 1-48. http://dx.doi.org/10.18637/jss.v067.i01.

Brandão, M. M., Vieira, F. A., Nazareno, A. G., \& Carvalho, D. (2015). Genetic diversity of neotropical tree Myrcia splendens (Myrtaceae) in a fragment-corridor system in the Atlantic rainforest. Flora, 216, 3541. http://dx.doi.org/10.1016/j.flora.2015.07.006.

Duarte, J. F., Carvalho, D., \& Vieira, F. A. (2015). Genetic conservation of Ficus bonijesulapensis R.M. Castro in a dry forest on limestone outcrops. Biochemical Systematics and Ecology, 59, 54-62. http://dx.doi.org/10.1016/j.bse.2015.01.008.

Falconer, D. S., \& MacKay, T. F. C. (1996). Introduction to quantitative genetics (464 p.). Harlow: Longman Group.

Faleiro, F. G., Andrade, S. R. M., \& Reis Junior, F. B. (2011). Biotecnologia: estado da arte e aplicações na agropecuária. Planaltina: Embrapa Cerrados.

Flores, T. B., Alvares, C. A., Souza, V. C., \& Stape, J. L. (2016). Eucalyptus no Brasil: zoneamento climático e guia para identificação (448 p.). Piracicaba: IPEF.

Henriques, E. P., Moraes, C., Sebbenn, A. M., Tomazello Filho, M., Moraes, M., \& Mori, E. S. (2016). Estimativa de parâmetros genéticos para caracteres silviculturais e densidade do lenho em teste de progênies de Eucalyptus urophylla. Scientia Forestalis, 44(109), 105-113. 
Higa, A. R., \& Carvalho, P. E. R. (1990). Sobrevivência e crescimento de doze espécies de eucalipto em Dois Vizinhos, Paraná. In Anais do VI Congresso Florestal Brasileiro (459 p.). São Paulo: Sociedade Brasileira de Silvicultura.

Instituto Agronômico do Paraná - IAPAR. (2019). Médias históricas em estações do IAPAR. Londrina: IAPAR. Recuperado em 30 de abril de 2019, de http://www.iapar.br/modules/conteudo/conteudo.php?conteudo=1070

Instituto de Pesquisas e Estudos Florestais - IPEF. (2018). Programa Cooperativo sobre Melhoramento Florestal. Piracicaba: IPEF. Recuperado em 16 de julho de 2018, de http://www.ipef.br/pcmf

Konzen, E. R., Navroski, M. C., Pereira, M. O., Nascimento, B., Meneguzzi, A., \& Souza, P. F. (2017). Genetic variation for growth variables of Eucalyptus benthamii Maiden et Cambage and E. smithii R. T. Baker provenances in southern Brazil. Cerne, 23(3), 359-366. http://dx.doi.org/10.1590/01047760201723032357.

Köppen, W., \& Geiger, R. (1928). Klimate der Erde. Gotha: Verlag Justus Perthes.

Lande, R. (1992). Neutral theory of quantitative genetic variance in a island model with local extinction and colonization. Evolution: International Journal of Organic Evolution, 46(2), 381-389. PMid:28564025. http://dx.doi.org/10.1111/j.1558-5646.1992.tb02046.x.

Massaro, R. A. M., Bonine, C. A. V., Scarpinati, E. A., \& Paula, R. C. (2010). Viabilidade de aplicação da seleção precoce em testes clonais de Eucalyptus spp. Ciência Florestal, 20(4), 597-609. http://dx.doi.org/10.5902/198050982418.

Miranda, A. C., de Moraes, M. L. T., Tambarussi, E. V., Furtado, E. L., Mori, E. S., Silva, P. H. M., \& Sebbenn, A. M. (2013). Heritability for resistance to Puccinia psidii Winter rust in Eucalyptus grandis Hill ex Maiden in Southwestern Brazil. Tree Genetics \& Genomes, 9(2), 321-329. http://dx.doi.org/10.1007/s11295-012-0572-x.

Moraes, C. B., Freitas, T. C. M., Pieroni, G. B., Zimback, L., \& Mori, E. S. (2011). Genetic variability in eucalypt for frost tolerance. In: IUFRO Working Group 2.08.03 improvement and of culture eucalyptus. Porto Seguro: IUFRO.

Moraes, C. B., Carvalho, E. V., Zimback, L., Luz, O. S. L., Pieroni, G. B., Mori, E. S., \& Leal, T. C. A. B. (2015) Variabilidade genética em progênies de meios-irmãos de eucaliptos para tolerância ao frio. Revista Árvore, 39(6), 1047-1054. http://dx.doi.org/10.1590/0100-67622015000600007.

Mori, E. S. (1988). Pomares de sementes florestais. Série Técnica IPEF, 5(16), 1-27.

Paiva, J. R., \& Valois, A. C. C. (2001). Espécies selvagens e sua utilização no melhoramento. In L. L. Nass, A. C. C. Valois, I. S. Melo \& M. C. Valadares-Inglis (Eds.), Recursos genéticos e melhoramento: plantas (pp. 79-99). Rondonópolis: Fundação MT.

Paludeto, J. G. Z., Estopa, R. A., \& Tambarussi, E. V. (2017). Eficiência da seleção precoce em clones de Eucalyptus grandis W. Hill ex Maiden x Eucalyptus urophylla S. T. Blake. Revista do Instituto Florestal, 29(2), 169-179. http://dx.doi.org/10.24278/2178-5031.201729203.

Paludzyszyn Filho, E., \& Santos, P. E. T. (2011). Programa de melhoramento genético de eucalipto da Embrapa Florestas: resultados e perspectivas. Brasília: Embrapa Florestas.

Pigato, S. M. P. C., \& Lopes, C. R. (2001). Caracterização silvicultural, botânica e avaliação da variabilidade genética por meio de marcador molecular RAPD em um teste de progênies de Eucalyptus urophylla S. T. Blake. Scientia Forestalis, (60), 135-148.

Pimentel-Gomes, F., \& Garcia, C. H. (2002). Estatística aplicada a experimentos agronômicos e florestais: exposição com exemplos e orientações para uso de aplicativos (309р.). Piracicaba: FEALQ.

Pires, I. E. (1996). Eficiência da seleção combinada no melhoramento genético de Eucalyptus spp (Tese de doutorado). Universidade Federal de Viçosa, Viçosa.

Pires, I. E., Resende, M. D. V., Silva, R. L., \& Resende Junior, M. F. R. (2011). Genética florestal (318 p.). Viçosa: Arka.

R Core Team. (2017). R: a language and environment for statistical computing. Vienna: R Foundation for Statistical Computing. Recuperado em 16 de julho de 2018, de https://www.R-project.org/

Resende, M. D. V. (1995). Delineamento de experimentos de seleção para maximização da acurácia seletiva e do progresso genético. Revista Árvore, 19(4), 479-500.

Resende, M. D. V. (2002). Genética biométrica e estatística no melhoramento de plantas perenes (975 p.). Brasília: Embrapa Informação Tecnológica.

Resende, M. D. V. (2007). Matemática e estatística na análise de experimentos e no melhoramento genético (561 p.). Colombo: Embrapa Florestas. 
Santos, G. A., Resende, M. D. V., Silva, L. D., Higa, A., \& Assis, T. F. (2013). Adaptabilidade de híbridos multiespécies de Eucalyptus ao Estado do Rio Grande do Sul. Revista Árvore, 37(4), 759-769. http://dx.doi.org/10.1590/S0100-67622013000400019.

Satterthwaite, F. E. (1946). An approximate distribution of estimates of variance components. Biometrics Bulletin, 2(6), 110-114. PMid:20287815. http://dx.doi.org/10.2307/3002019.

Sebbenn, A. M., \& Ettori, L. C. (2001). Conservação genética ex situ de Esenbeckia leiocarpa, Myracrodruon urundeuva e Peltophorum dubium em teste de progênie misto. Revista do Instituto Florestal, 13(2), 201-211.

Sebbenn, A. M., Freitas, M. L. M., Zanatto, A. C. S., Moraes, E., \& Moraes, M. A. (2007). Conservação ex situ e pomar de sementes em banco de germoplasma de Balfourodendron riedelianum. Revista do Instituto Florestal, 19(2), 101-112.

Silva, P. H. M., Paula, R. C., \& Moraes, M. L. T. (2018). Melhoramento de populações de eucaliptos (1. ed., 108 p.). Piracicaba: IPEF.

Spitze, K. (1993). Population structure in Daphnia obtusa: quantitative genetic and allozymic variation. Genetics, 135(2), 367-374. PMid:8244001.

Sturion, J. A. (1993). Variação genética de características de crescimento e de qualidade da madeira em progênies de Eucalyptus viminalis Labill (Tese de doutorado). Universidade Federal do Paraná, Curitiba.

Tambarussi, E. V., Lima, B. M., Queiroz, R. C., Peres, F. S. B., Dias, D. C., Pagliarini, M. K., Pereira, F. B., Rosa, J. R. B. F., \& Rezende, G. D. S. P. (2017). Estimativa de parâmetros genéticos para a seleção precoce em clones de Eucalyptus spp. Scientia Forestalis, 45(115), 507-517. http://dx.doi.org/10.18671/scifor.v45n115.08.

Vencovsky, R. (1987). Tamanho efetivo populacional na coleta e preservação de germoplasma de espécies alógamas. IPEF, (35), 79-84.

Whitlock, M. C. (2008). Evolutionary inference from Molecular Ecology, 17(8), 1885-1896. PMid:18363667. http://dx.doi.org/10.1111/j.1365-294X.2008.03712.x.

Contribuição dos autores: JGZP: conceituação, curadoria de dados; MP: curadoria de dados, escrita - primeira redação, escrita - revisão e edição; LVM: Análise Formal, Investigação, Metodologia, Escrita - Primeira Redação; JRMS: escrita - primeira redação, VAP: visualização, escrita - primeira redação; EVT: administração do projeto, conceituação, escrita - revisão e edição. 